\title{
Erratum to "Poincaré renormalized forms"
}

\author{
Giuseppe Gaeta
}

The computation given in section 12 of [1] is incorrect for the case $\nu \leq \mu$ (these are defined in Lemma 2 there). The correct expression for formula (12.14) in Lemma 2 is

$$
f^{*}(x)=A x+a_{\mu} X_{\mu}+\widetilde{a}_{2 \mu} X_{2 \mu}+\sum_{k=\nu}^{\mu} \widetilde{b}_{k} Y_{k}
$$

where $\widetilde{a}_{k}, \widetilde{b}_{k}$ are real constants, in general different from $a_{k}, b_{k}$.

Note also that in [1] it is not sufficiently stressed that this computation is performed by making use of the Lie algebraic properties of the set (12.2) of vector fields in normal form. Following step by step the general and generic algorithm described in sections $6-9$ one would obtain a different reduced normal form, i.e. $f^{*}(x)=A x+b_{\nu} Y_{\nu}+\sum_{k=\mu}^{\infty} \widetilde{a}_{k} X_{k}$.

Similar considerations and corrections also apply to sect.13 of [1] and sect. VIII.6 of [2].

I stress that in [3] my definition of PRFs was incorrectly reported; thus the remarks given there do not concern PRFs.

These points, and the related computations, are discussed in detail in [4], available via $h t t p: / / m p e j . u n i g e . c h / m p \_a r c / m p \_a r c-h o m e . h t m l$.

\section{References}

[1] G. Gaeta, "Poincaré renormalized forms", Ann. Inst. H. Poincaré (Phys. Theo.) 70 (1999), 461-514.

[2] G. Cicogna and G. Gaeta, Symmetry and perturbation theory in nonlinear dynamics, Springer (Lecture Notes in Physics, vol. m57), 1999.

[3] A.D. Bruno, reviews 1999a:34111 and 2000h:37071, Mathematical Reviews.

[4] G. Gaeta, "Poincaré renormalized forms and regular singular points of vector fields in the plane", preprint mp-arc 01-17 (2001); "Algorithmic reduction of Poincaré normal forms and Lie algebras", in preparation.

Giuseppe Gaeta

Dipartimento di Fisica

Università di Roma

I-00185 Roma, Italy

Communicated by Vincent Rivasseau

received 05/02/01 